\title{
Al doping effect on magnetic phase transitions of magnetoelectric hexaferrite $\mathrm{Ba}_{0.7} \mathrm{Sr}_{1.3} \mathrm{Zn}_{2}\left(\mathrm{Fe}_{1-x} \mathrm{Al}_{x}\right)_{12} \mathrm{O}_{22}$

\author{
Hun Chang, ${ }_{1}^{1}$ Hak Bong Lee, ${ }^{1}$ Young-Sang Song, ${ }^{1}$ Jae-Ho Chung, ${ }^{1,}$ S. A. Kim, ${ }^{2}$ I. H. Oh, ${ }^{2}$ M. Reehuis, ${ }^{3}$ and J. Schefer ${ }^{4}$ \\ ${ }^{1}$ Department of Physics, Korea University, Seoul 136-713, South Korea \\ ${ }^{2}$ Neutron Science Division, Korea Atomic Energy Research Institute, Daejeon, South Korea \\ ${ }^{3}$ Helmholtz-Zentrum für Materialien und Energie, D-14109 Berlin, Germany \\ ${ }^{4}$ Laboratory for Neutron Scattering, Paul Scherrer Institute, CH-5232 Villigen PSI, Switzerland
}

(Received 10 November 2011; published 1 February 2012)

\begin{abstract}
We investigated the effect of $\mathrm{Al}$ doping in magnetic properties of the Y-type hexaferrite $\mathrm{Ba}_{0.7} \mathrm{Sr}_{1.3} \mathrm{Zn}_{2}\left(\mathrm{Fe}_{1-x} \mathrm{Al}_{x}\right)_{12} \mathrm{O}_{22}(0 \leqslant x \leqslant 0.12)$, which exhibit field-induced magnetoelectric polarization. We find that $\mathrm{Al}$ doping increases the pitch of a spin helix and enhances $c$-axis magnetization, stabilizing longitudinal conical phases. These conical phases eventually collapse at $x \geqslant 0.10$. These results suggest that competitions between easy-axis and easy-plane anisotropy fields play a key role in generating stable magnetoelectric polarization in Y-type hexaferrites.
\end{abstract}

DOI: 10.1103/PhysRevB.85.064402

PACS number(s): 75.85.+t, 75.25.-j, 75.30.Gw, 75.30.Kz

\section{INTRODUCTION}

Recent observations of magnetoelectric polarization in a series of hexagonal ferrites demonstrate promising outlooks for realizing practical applications of the novel multiferroic effect. ${ }^{1-7}$ The key ingredient of the magnetoelectricity is spin-driven ferroelectric polarization arising due to anisotropic coupling between spin and orbital degrees of freedom..$^{9-11}$ It is now fairly well understood that magnetic ordering with broken inversion symmetry is essential in producing net electric polarization., ${ }^{9}{ }^{12-18}$ Early experimental efforts were focused on establishing magnetic control of the ferroelectric polarization, ${ }^{19,20}$ whereas lately more attention has been given to electric control of magnetic order in bulk or thin films. ${ }^{21-24}$ One of the biggest challenges is to realize the strong coupling at ambient temperature, because the effect is often observed at cryogenic temperatures. Hexagonal ferrites are already providing evidence of magnetoelectric coupling robust at room temperature (RT) based on strong superexchange interactions between $\mathrm{Fe}$ ions. ${ }^{5-8}$

Ferrites with a hexagonal lattice have long been studied due to their diverse magnetic properties, which are stable at RT and above. ${ }^{25}$ Among them, Y-type hexaferrites are the most widely studied in terms of magnetoelectricity. ${ }^{1-3,26-28}$ Their crystal structures belong to $R \overline{3} m$ and have fairly long $c$-axis parameters $(\approx 43.56 \AA)$ in the hexagonal setting. Magnetic $\mathrm{Fe}^{3+}\left(S=\frac{5}{2}\right)$ ions are located inside either oxygen tetrahedra or octahedra, which then form a series of layers stacked along the $c$ axis. The densely packed magnetic layers are typically grouped into large (L) and small (S) spin blocks. [See Fig. 1(a).] Within each block, spin vectors are nearly collinear. Superexchange interactions between these layers are responsible not only for setting up long-range magnetic order above RT, but also establishing stable incommensurate helical ordering in $\mathrm{Ba} / \mathrm{Sr}$-mixed compounds. ${ }^{29-31}$ For instance, $\mathrm{Ba}_{0.5} \mathrm{Sr}_{1.5} \mathrm{Zn}_{2} \mathrm{Fe}_{12} \mathrm{O}_{22}$ (BSZFO) undergoes an antiferromagnetic (AFM) phase transition at $T_{N}=337 \mathrm{~K}$, followed by an incommensurate planar helix $(\mathrm{PH})$ below $310 \mathrm{~K}$. [See Fig. 1(b).] The PH ground state suggests a significant easyplane magnetocrystalline anisotropy, which confines the spin vectors close to the $a b$ planes. ${ }^{32}$ Electric polarization, however, is not observed in the $\mathrm{PH}$ phase because spin-current vectors remain parallel to the axis of the spin helix. ${ }^{9}$ Finite polarization appears only when a sufficiently large external field $\left(\mu_{0} H \gtrsim\right.$ $0.3 \mathrm{~T}$ ) is applied perpendicular to the $c$ axis. ${ }^{1}$

The magnetoelectricity in Y-type hexaferrites can be enhanced by judiciously replacing cations. The most recent works have reported that the onset fields were lowered by several orders of magnitudes in $\mathrm{Ba}_{2} \mathrm{Mg}_{2} \mathrm{Fe}_{12} \mathrm{O}_{22}$ (BMFO) or $\mathrm{Ba}_{0.5} \mathrm{Sr}_{1.5} \mathrm{Zn}_{2}\left(\mathrm{Fe}_{1-x} \mathrm{Al}_{x}\right)_{12} \mathrm{O}_{22}\left(\mathrm{Al}_{x}-\mathrm{BSZFO}\right){ }^{2,23}$ It is believed that the cation doping reduces magnetocrystalline anisotropy, which eventually leads to lowering of the onset fields. In this picture, a reduction of easy-plane anisotropy results in the appearance of axial spin components along the $c$ axis, and subsequently stabilizes longitudinal conical (LC) spin structures. ${ }^{28}$ [See Fig. 1(c).] Then finite magnetoelectric polarization can easily appear when the LC configuration flops into a transverse orientation under small field. ${ }^{2}$ It remains unclear, however, how the LC structure stabilizes upon reduction of the easy-plane anisotropy. This is because planar spin configurations will still minimize the total magnetic energy that includes isotropic exchanges and single-ion anisotropy terms, regardless of the magnitude of the easy-plane anisotropy. Interestingly, neutron diffraction measurements also revealed that spin ordering under an external field is not incommensurate, but commensurate to the lattice with $90^{\circ}$ half-pitch between neighboring spin blocks. ${ }^{26-28,32}$ Such an angle is unnatural in superexchange-driven antiferromagnets, and thus suggests that one should consider more than easyplane anisotropy in order to understand the magnetoelectric properties of the Y-type hexaferrites.

In this work, we have performed systematic investigations of the magnetic phase transitions in $\mathrm{Al}_{x}-\mathrm{BSZFO}$ (or Al-doped $\mathrm{BSZFO}$ ) as a function of $\mathrm{Al}$ concentration below $400 \mathrm{~K}$. Our primary interest is in understanding the role of $\mathrm{Al}$ dopants, and particularly how they change magnetocrystalline anisotropy and lead to the formation of the LC phases. Experimental observations of single crystals confirmed that Al doping allows magnetic moments to be relieved of the $\mathrm{PH}$ ordering and develop LC structures. When $\mathrm{Al}$ doping exceeds $10 \%$ 
(a)

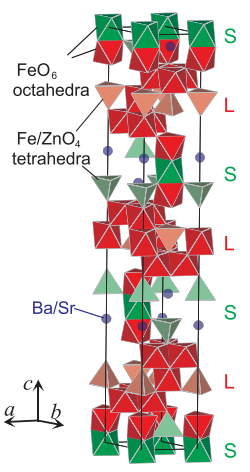

$\begin{array}{ll}\text { (b) } x=0.0 & \text { (c) } x=0.08\end{array}$

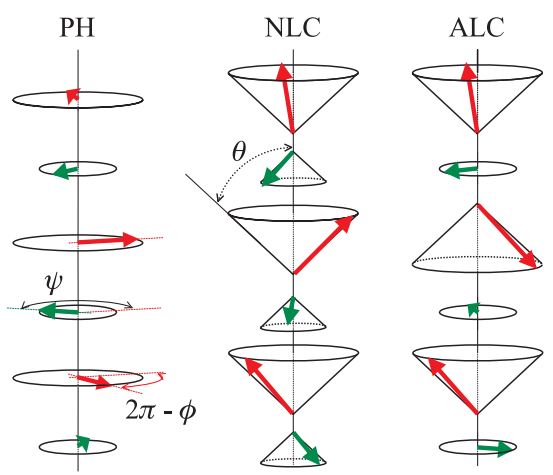

FIG. 1. (Color online) (a) Crystallographic unit cell of Y-type hexaferrite lattice. Red and green colors indicate L and S blocks, respectively. (b) Simplified representation of the planar helical spin structure of Al-free BSZFO. (c) Normal longitudinal and alternating longitudinal conical spin structures of $\mathrm{Al}_{0.08}$-BSZFO. Red and green arrows indicate net magnetization vectors of $\mathrm{L}$ and $\mathrm{S}$ blocks, respectively.

(or $x \geqslant 0.10$ ), the LC phases finally collapse into commensurate order, which coincides with the previously reported deterioration of magnetoelectric susceptibility. ${ }^{3}$ Using the simple model based on superexchange interaction between the $\mathrm{L}$ and $\mathrm{S}$ blocks, ${ }^{33}$ we argue that competition between easy-axis and easy-plane anisotropy fields plays an important role in stabilizing the LC ordering.

\section{EXPERIMENTAL}

Single-crystalline samples were grown using the $\mathrm{Na}_{2} \mathrm{O}$ $\mathrm{Fe}_{2} \mathrm{O}_{3}$ flux. ${ }^{34}$ Starting materials $\left(\mathrm{BaCO}_{3}, \mathrm{SrCO}_{3}, \mathrm{ZnO}, \mathrm{Fe}_{2} \mathrm{O}_{3}\right.$, $\mathrm{Al}_{2} \mathrm{O}_{3}$, and $\mathrm{Na}_{2} \mathrm{CO}_{3}$ ) were carefully weighed and thoroughly mixed in agate mortars. The mixture was then placed in a $\mathrm{Pt}$ crucible and heated slowly in an electric furnace. It was then held at $1450{ }^{\circ} \mathrm{C}$ for $12 \mathrm{~h}$ and slowly cooled at the rate of $1{ }^{\circ} \mathrm{C} / \mathrm{h}$ down to $700{ }^{\circ} \mathrm{C}$. The obtained crystals typically had hexagonal platelet geometry, in which the largest surfaces correspond to $a b$ planes. Although starting mixtures were made for the ratio $\mathrm{Ba}: \mathrm{Sr}=0.5: 1.5$, electron probe microanalysis (EPMA) showed that the ratios in our crystals were typically close to 0.7:1.3. Previous works show that the pitch of the $\mathrm{PH}$ phases only differs by less than $10 \%$ between the two compositions. ${ }^{30}$ We also note that electric polarizations in our samples (not shown) are observed over nearly identical field ranges to those reported for $\mathrm{Ba}: \mathrm{Sr}=0.5: 1.5,{ }^{3}$ whereas the magnitude of the polarization is smaller in our samples by an order of magnitude.

dc magnetization measurements were performed using a commercial vibrating sample magnetometer below $400 \mathrm{~K}$ with magnetic field applied parallel or perpendicular to the $c$ axis. Neutron diffraction measurements were performed using three four-circle diffractometers. The majority of data were collected using the FCD diffractometer $(\lambda=1.31 \AA)$ of the Korea Atomic Energy Research Institute. The data for $x=0.04$ and 0.08 were collected using the TriCS (Ref. 35) $[\lambda=2.32(1) \AA]$ at the Swiss Spallation Source (SINQ) (Ref. 36) of the Paul Scherrer Institute and the E5 $(\lambda=$ $2.38 \AA$ ) of Helmholtz-Zentrum Berlin (HZB) für Materialien und Energie, respectively. In all measurements, single-crystal

samples were glued to thin vanadium rods, which were then mounted in He-filled aluminum containers. The temperature during the diffraction measurements was controlled using a closed-cycle He refrigerator between 10 and $300 \mathrm{~K}$.

\section{RESULTS AND DISCUSSIONS}

\section{A. Overview of the Al doping effect}

Previous works reported an AFM transition in Al-free BSZFO with $\mathrm{Ba}: \mathrm{Sr}=0.5: 1.5$ at $T_{N}=337 \mathrm{~K}$, below which spin vectors are lying in the $a b$ planes. $^{31}$ A consistent transition is observed at the same temperature in our data as $\lambda$ anomalies of the low-field magnetization, or $M(T)$, curves. [See the data labeled $x=0.00$ in Figs. 2(a) and 2(b).] At lower temperatures below the anomaly, the $M(T)$ curve grows strongly within the $a b$ planes but remains suppressed along the $c$ axis. These behaviors are consistent with the spin vectors confined in the $a b$ planes due to easy-plane anisotropy, which thus respond weakly to the field perpendicular to the planes. We also note that, although the $M(T)$ curves in Fig. 2 do

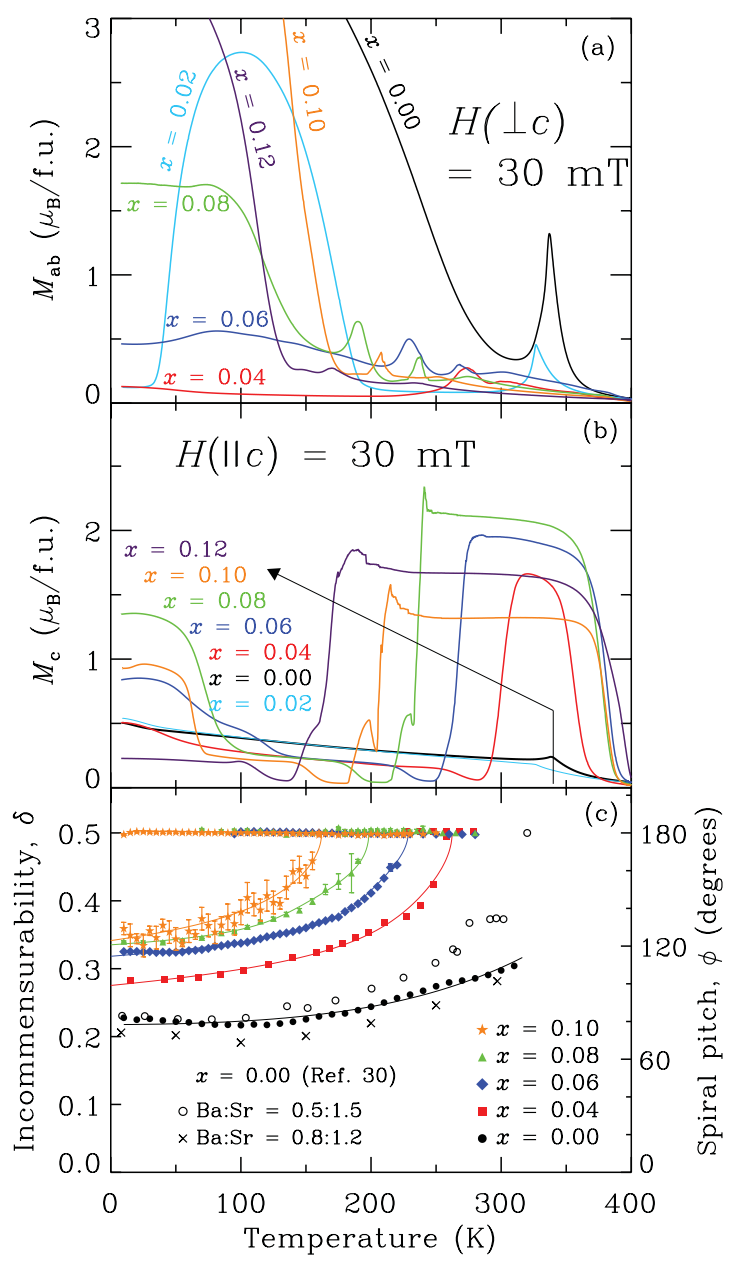

FIG. 2. (Color online) Temperature and doping dependences of low-field magnetization $\left(\mu_{0} H=30 \mathrm{mT}\right)$ under external fields along (a) $H \perp c$ and (b) $H \| c$. Shown in (c) is the incommensurability $(\delta)$ of the helical or conical ordering observed by neutron diffraction. It is directly related to the spiral pitch $(\phi)$ between net magnetization vectors of the nearest L (or S) blocks. [See Fig. 1(b).] 
not reveal an anomaly associated with the commensurate-toincommensurate transition at $310 \mathrm{~K},{ }^{31}$ a signature is clearly observed in $M(H)$ curves (not shown).

When a small amount of $\mathrm{Al}$ doping is introduced, interesting changes are observed along both directions. Figure 2 shows that the $\lambda$ anomaly of the low-field magnetization is shifted to a slightly lower temperature for $x=0.02$. We also notice that $M_{a b}(T)$ is suddenly suppressed below $\leqslant 50 \mathrm{~K}$ whereas $M_{c}(T)$ is slightly enhanced. This indicates that a weak $c$-axis magnetization begins to build up at low temperatures. For $x=0.04$, the $\lambda$ anomaly is suddenly replaced by a strong plateau in $M_{c}(T)$, whereas $M_{a b}(T)$ is fully suppressed below RT. Interestingly, the onset of the plateau is observed at a higher temperature near $350 \mathrm{~K}$. The temperature range for the plateau continues to expand in both directions with an increasing amount of $\mathrm{Al}$ doping. We notice that $M_{c}(T)$ curves typically reach their minima just below the plateau but exhibit large enhancements upon further cooling. Their low- $T$ enhancement reaches the maximum at $x=0.08$, where the largest magnetoelectric susceptibility has been reported. ${ }^{3}$ This behavior is ascribed to the appearance of the normal longitudinal conical (NLC) phase. ${ }^{28}$ [See Fig. 1(c).] We also notice a few weak features in the intermediate temperature range, indicating additional magnetic phase transitions. For instance, the weak enhancement in $M_{c}(T)$ just below its minimum coincides with a transition into the alternating longitudinal conical (ALC) phase, in which the commensurate axis components alternate along the $c$ axis. ${ }^{28}$ [See Fig. 1(c).]

As shown in Fig. 2(c), it is at the onset of the ALC phase where the incommensurate order $\left[\boldsymbol{k}_{2}=(0,0,3 \delta)\right.$ (Ref. 37)] begins to be observed by neutron diffraction. Temperature dependences of the neutron diffraction intensities are displayed in Fig. 3, which are in good agreement with the magnetization data. Note that only the intensities appearing at wave vectors satisfying the condition $-h+k+l=3 n$ are due to structural

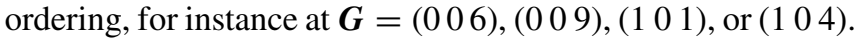
In Fig. 3, all the intensities at other wave vectors are purely due to magnetic ordering. In particular, the bell-shaped distribution of intensities accounts for incommensurate $\boldsymbol{k}_{2}$ order, such that $\boldsymbol{Q}_{M}=\boldsymbol{G} \pm \boldsymbol{k}_{2}$. For $x=0.0$, the incommensurability gradually changes from $\delta \approx 0.2$ at $10 \mathrm{~K}$ to $\delta \approx 0.3$ near RT, which can directly be converted to the pitch of the helix by the relation $\phi=2 \pi \delta$. [See Fig. 2(c).]

Similar incommensurate wave vectors continue to be observed in Al-doped BSZFO up to $x=0.08$, but their temperature ranges gradually shrink. In $\mathrm{Al}_{0.04}$-BSZFO, the two incommensurate peaks [e.g., at $\left(\begin{array}{lll}0 & 0 & 6\end{array}\right)+\boldsymbol{k}_{2}$ and $\left(\begin{array}{lll}0 & 0 & 9\end{array}\right)-\boldsymbol{k}_{2}$ ] merge into a single commensurate peak [e.g., at $\left(\begin{array}{lll}0 & 0 & 6\end{array}\right)+\boldsymbol{k}_{1}$, where $\left.\boldsymbol{k}_{1}=(0,0,3 / 2)\right]$ at $265 \mathrm{~K}$, similarly to what has been reported for $x=0.0$ at $310 \mathrm{~K}^{31}$ [See Fig. 3(b).] The diffraction intensity observed along $\left[\begin{array}{ll}1 & 0 l\end{array}\right]$, however, reveals an additional transition that is not observable along $\left[\begin{array}{lll}0 & 0 & l\end{array}\right]$. For instance, the intensity at $(105 / 2)$ persists well below $265 \mathrm{~K}$, indicating the coexistence of $\boldsymbol{k}_{1}$ and $\boldsymbol{k}_{2}$. Note that the corresponding intensity is missing at (0 $015 / 2)$, suggesting that the associated spin vectors are parallel to the $c$ axis. The coexistence of the two propagation vectors is thus a signature that the ALC phase exists in this temperature range for $x=0.04$. The temperature range for the ALC phase widens but shifts down to 70-180 K for $x=0.08$. [See Fig. 3(c).] Below this temperature range, a

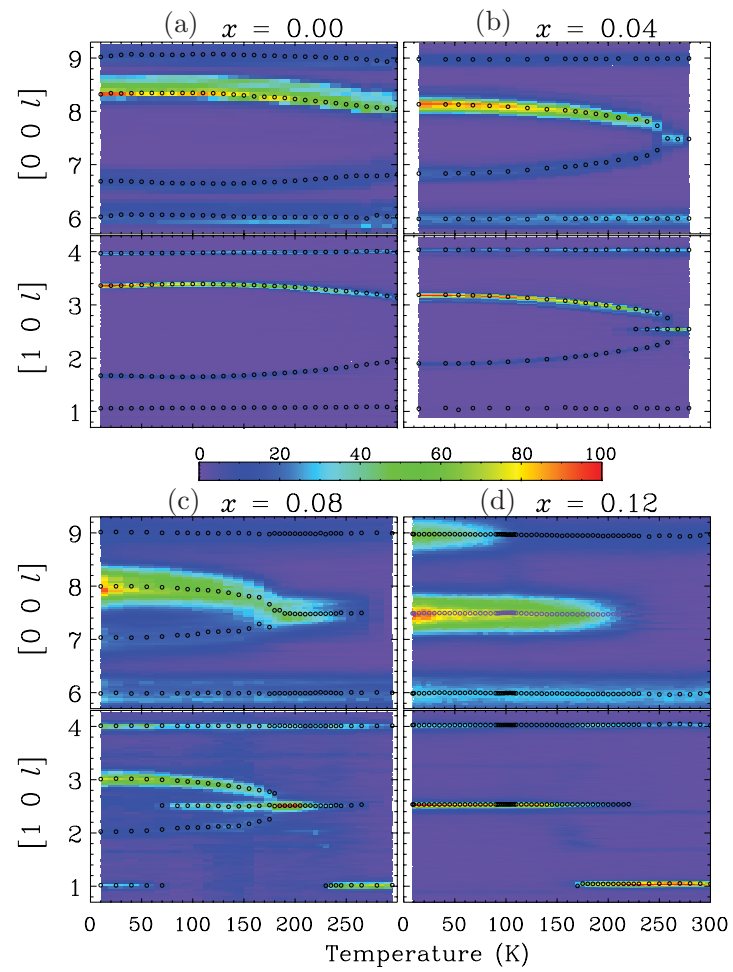

FIG. 3. (Color online) Temperature dependence of the neutron diffraction intensities collected along two directions in $Q$ space: [0 $\left.\begin{array}{ll}0 & l\end{array}\right]$ and $\left[\begin{array}{lll}1 & 0 & l\end{array}\right]$ for (a) $x=0.00$, (b) $x=0.04$, (c) $x=0.08$, and (d) $x=0.12$. Empty circles mark peak positions fitted by Gaussians.

strong magnetic intensity appears at $\left(\begin{array}{lll}1 & 0 & 1\end{array}\right)$, which indicates a ferrimagnetic (FIM) order $\left[\boldsymbol{k}_{0}=(0,0,0)\right]$. The coexistence of $\boldsymbol{k}_{0}$ and $\boldsymbol{k}_{2}$ accounts for the NLC phase. ${ }^{28}$

The incommensurate peaks disappear when the amount of Al doping exceeds $10 \%$, indicating that the conical phases no longer exist. Figure $3(\mathrm{~d})$ shows that, in $\mathrm{Al}_{0.12}$-BSZFO, the $\boldsymbol{k}_{1}$ peaks instead are strongly present along both directions below $220 \mathrm{~K}$. We also notice that intensities due to $\boldsymbol{k}_{0}$ still appear but along unexpected directions below $120 \mathrm{~K}$. Magnetocrystalline anisotropy is certainly compromised due to heavy doping. The magnetoelectric susceptibility of Al-doped BSZFO has been reported to reach the maximum at $x=0.08$, beyond which it quickly deteriorates. ${ }^{3}$ The current results indeed suggest that the doping dependence of the magnetoelectric property is closely tied to the evolution of magnetic structure. We thus classify Al-doped BSZFO into three regimes: underdoped for $x \leqslant 0.04$, optimally doped for $0.04<x \leqslant 0.08$, and overdoped for $0.08<x$.

\section{B. Underdoped $(x \leqslant 0.04)$}

Summarized in Fig. 4 are the data measured by the vibrating sample magnetometry and the neutron diffraction for $x=0.0$ and 0.04 . The upper two panels show the remanent magnetization, $M(0)$, and the temperature derivative of lowfield magnetization, $d M / d T$, along the two directions. The remaining panels show the integrated intensities of nuclear and magnetic Bragg peaks. Since the neutron scattering measurements were limited to below $300 \mathrm{~K}$, the magnetization 


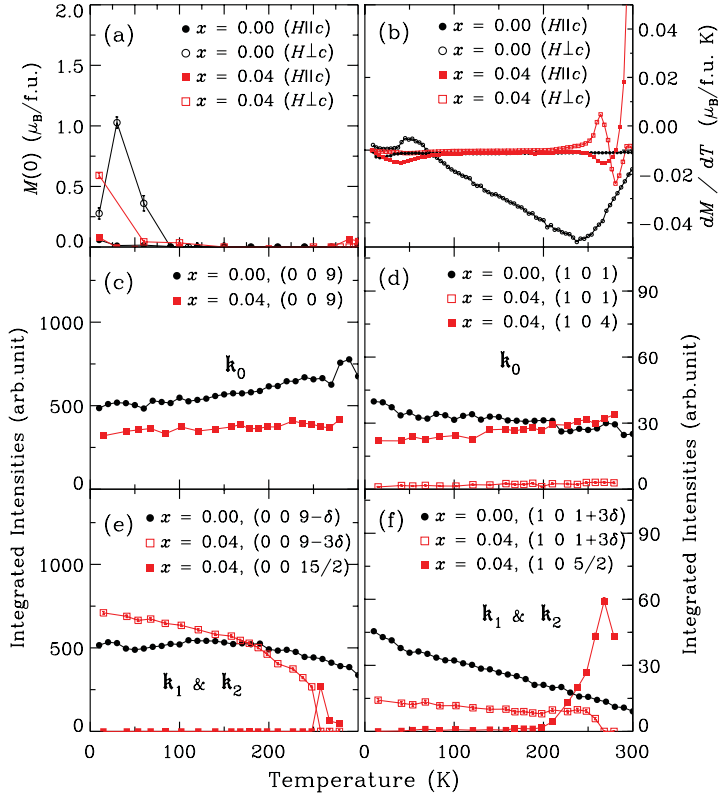

FIG. 4. (Color online) Temperature dependences for the underdoped BSZFO samples of (a) the remanent magnetization, (b) temperature derivative of low-field magnetization, and (c)(f) integrated neutron diffraction intensities of Bragg peaks. The integrated intensities were obtained by fitting Gaussian peaks to the data collect by $l$ scans. ${ }^{38}$

data are also presented for the same temperature range only. In the case of Al-free BSZFO, we do not observe any significant temperature-dependent changes below $300 \mathrm{~K}$. Only weak features in $d M_{a b} / d T$ are observed near $50 \mathrm{~K}$. Neutron diffraction intensities show monotonic dependence only, suggesting that underlying spin structures hardly change. The apparent large values of $d M_{a b} / d T$ are ascribed to the spins easily moving within the $a b$ planes, but are not associated with any additional phase transitions.

The data for $x=0.04$ exhibit sharp changes at higher temperatures, indicating FIM order, but they are relatively featureless below $200 \mathrm{~K}$. The intensities of the nuclear Bragg peaks do not show any significant changes either. In fact, all the samples in the underdoped regime show small finite $M_{c}(0)$ below $30 \mathrm{~K}$, typically less than $0.1 \mu_{B}$ per formula unit. Transitions into this range are also visible as small downward turns of $d M_{c} / d T$ curves near $50 \mathrm{~K}$ or below, commonly observed for all underdoped samples. We suspect, therefore, that weak LC order already exists in this regime. Their low- $T$ behaviors appear probably because the anisotropy minima are slightly tilted away from the $a b$ planes. ${ }^{29}$ Nevertheless, the $c$-axis magnetization, if any, must be too small to be detected by neutron diffraction. The ALC phase, in contrast, clearly appears between 200 and $270 \mathrm{~K}$. The maximum of $d M_{a b} / d T$ at $\approx 270 \mathrm{~K}$ coincides with the onset of $\boldsymbol{k}_{2}$ and the maximization of $\boldsymbol{k}_{1}$, indicating the formation of the ALC phase.

\section{Optimally doped $(x=0.06$ and 0.08$)$}

The data for $x=0.06$ and 0.08 are summarized in Fig. 5 . They reveal several features that are in clear contrast to the undoped group. Above all, there is a large enhancement in remanent magnetization along the $c$ axis at low temperatures,

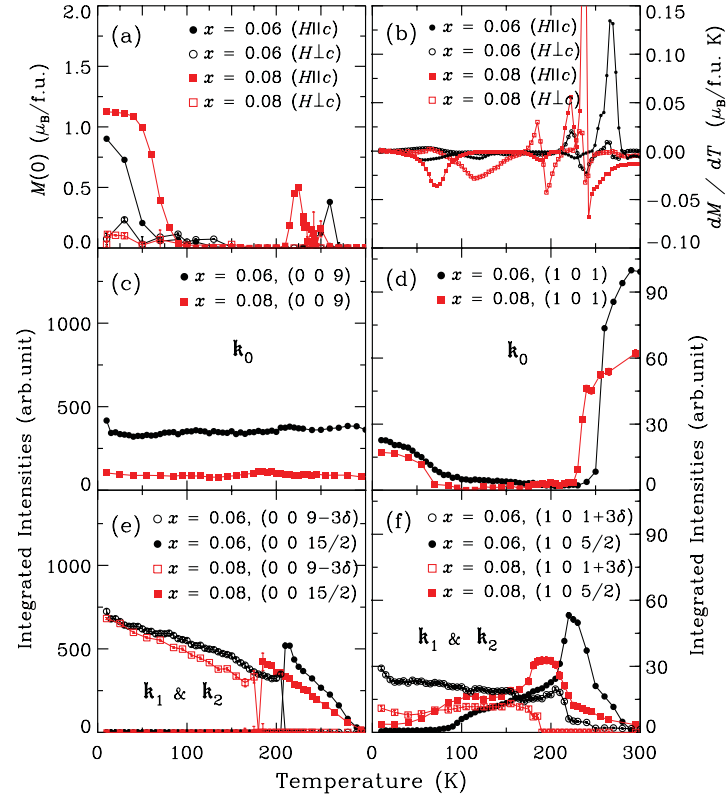

FIG. 5. (Color online) Temperature dependences for the optimally doped BSZFO samples of (a) the remanent magnetization, (b) temperature derivative of low-field magnetization, and (c)-(f) integrated neutron diffraction intensities of Bragg peaks. ${ }^{38}$

which directly overlaps with the increase in diffraction intensities of nuclear Bragg peaks. These two data consistently account for the cone axis of the LNC phase. ${ }^{28}$ Note that the downward turn of the $d M_{c} / d T$ curves observed at this transition is similar to the low- $T$ behavior of the undoped group. At high temperatures, on the other hand, $\left(\begin{array}{lll}1 & 0 & 1\end{array}\right)$ peak intensities show large increases associated with the FIM ordering, which already appeared for $x=0.04$. The transition into this phase is clearly observed as sharp peaks in $d M_{c} / d T$ curves, too. It is important to realize, however, that the remanent magnetization is absent in this FIM phase. Their field response is very soft, although spin vectors in this phase are aligned parallel to the $c$ axis.

The ALC phase also continues to expand its temperature range. The $\mathrm{PH}$ phase is visible for $x=0.06$ only briefly as a small step in $M_{c}(T)$, but absent for $x=0.08$. Instead, the NLC phase transforms directly into the ALC phase. Neutron diffraction data clearly show that $\boldsymbol{k}_{1}$ and $\boldsymbol{k}_{2}$ peaks coexist in the ALC phase. The $\boldsymbol{k}_{2}$ wave vector finally merges to $\boldsymbol{k}_{1}$ at $190 \mathrm{~K}$, which coincides with the minimization of $M_{c}(T) . \mathrm{Al}_{0.06}$-BSZFO also shows similar behaviors in most of the observed temperature range. We also notice another brief appearance of finite $M_{c}(T)$ at $215-240 \mathrm{~K}$ for $x=0.08$ (or $245-270 \mathrm{~K}$ for $x=0.06$ ). Magnetic diffraction intensities in this range appear at unexpected positions, for instance at (1 0 7/4). The associated magnetic structures are not yet fully understood, and will be discussed in separate publications.

\section{Overdoped $(0.10 \leqslant x)$}

The data for $x=0.10$ and 0.12 are summarized in Fig. 6. In this overdoped regime, incommensurate $\boldsymbol{k}_{2}$ intensities almost 


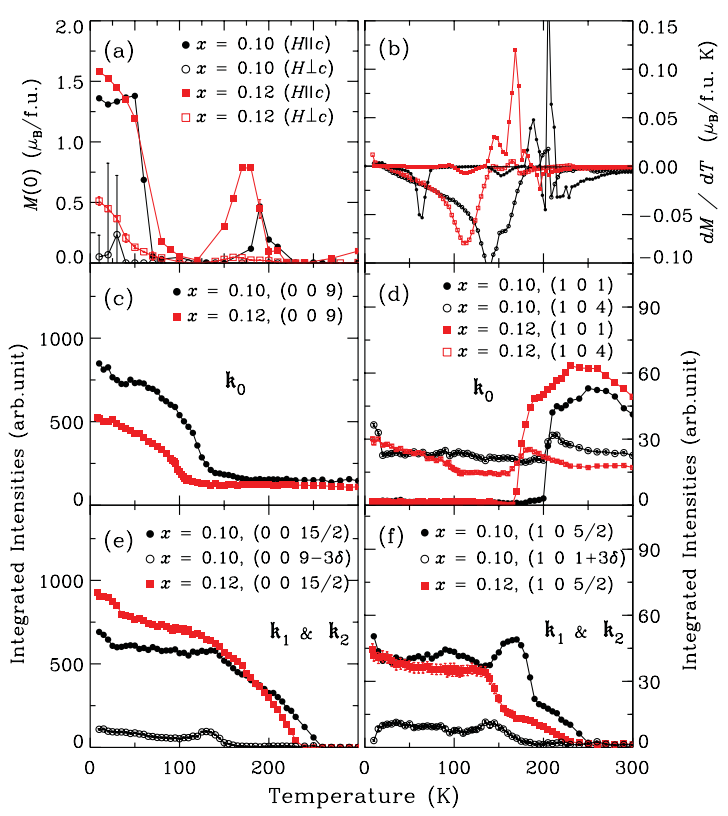

FIG. 6. (Color online) Temperature dependences for the overdoped BSZFO samples of (a) the remanent magnetization, (b) the temperature derivative of low-field magnetization, and (c)-(f) integrated neutron diffraction intensities of Bragg peaks. ${ }^{38}$

disappear. Momentum dependence of the neutron diffraction intensities (not shown) shows very weak and broad intensities for $x=0.10$, indicating that the incommensurate order is only marginally stable in this composition. These intensities completely vanish in $\mathrm{Al}_{0.12}$-BSZFO. Instead, commensurate $\boldsymbol{k}_{1}$ peaks prevail at low temperatures. Another noticeable behavior for $x=0.12$ at low temperatures is that both $\boldsymbol{k}_{0}$ and $\boldsymbol{k}_{1}$ peaks are observed along both [ $\left[\begin{array}{lll}0 & 0 & l\end{array}\right]$ and [ $\left[\begin{array}{lll}1 & 0 & l\end{array}\right]$ directions. This suggests that an underlying spin arrangement is no longer tied to high-symmetry directions. Finite remanent magnetization observed along both directions also supports this idea. The overall behavior of the overdoped regime indicates that the magnetocrystalline anisotropy is largely compromised due to heavy $\mathrm{Al}$ doping.

\section{E. The phase diagram}

Using the transition temperatures observed from the magnetization and the neutron diffraction measurements, we have mapped out the $x$ - $T$ phase diagram of $\mathrm{Al}_{x}$-BSZFO below $400 \mathrm{~K}$ and shown it in Fig. 7. Simplified spin structures of each magnetic phase are listed in Fig. 8. They provide a very clear view that the incommensurate $\boldsymbol{k}_{2}$ ordering is gradually overtaken by $\boldsymbol{k}_{0}$ and $\boldsymbol{k}_{1}$ ordering. The Al doping effect can be summarized to be working in two directions. First, it suppresses incommensurate spin ordering. Second, it enhances spin components along the $c$ axis. The former is more closely related to exchange interactions, whereas the latter is probably related to the magnetocrystalline anisotropy. Previous works report that doped $\mathrm{Al}$ ions preferentially substitute $\mathrm{Fe}$ ions in the octahedral sites. ${ }^{3}$ Given the structure of a corundum, it is natural for $\mathrm{Al}^{3+}$ ions to prefer six-oxygen coordination. Therefore, it is reasonable to assume that Al doping will effectively reduce the magnetization of the octahedral sites

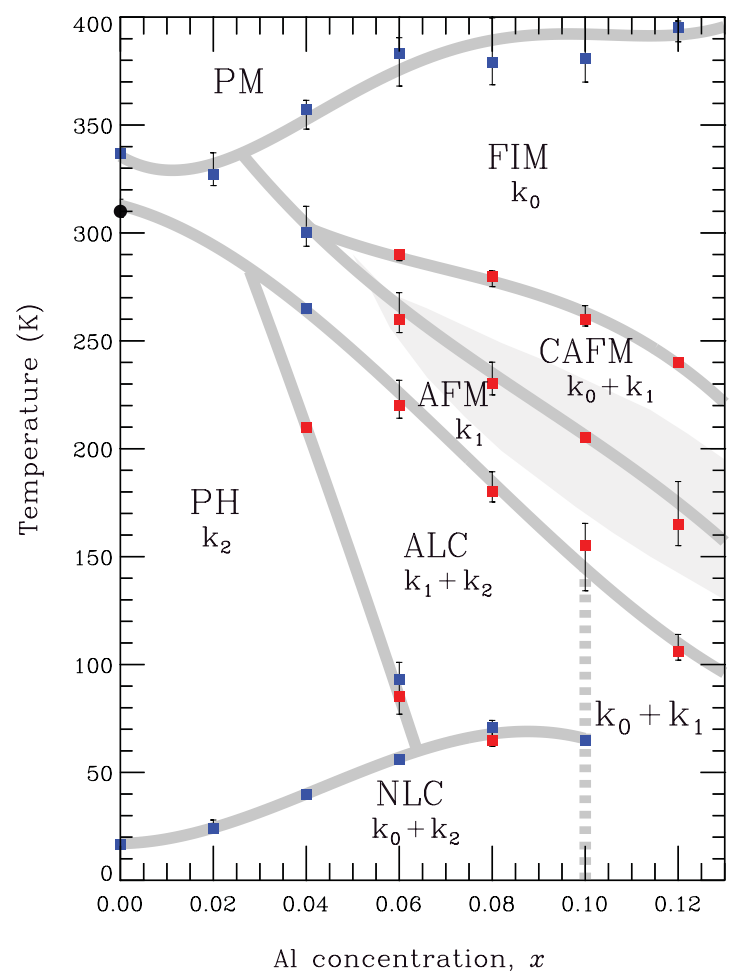

FIG. 7. (Color online) The $x$-T phase diagram of Al-doped BSZFO. The blue and red squares are obtained from magnetization and neutron diffraction data, respectively. The solid circle is from Ref. 31. The shaded area marks the unknown phase discussed in the text.

while not significantly affecting that of the tetrahedral sites. It is then natural to expect reductions both in exchange interactions and anisotropy fields due to the octahedral ions.

Magnetocrystalline anisotropy on Fe sites may arise due to couplings between spin and orbital parts of the electronic angular momentum, particularly when the local crystallographic environment endorses finite expectation values of the orbital momenta. Each $\mathrm{FeO}_{4}$ tetrahedron in BSZFO has one of its vertices along the $c$ axis, whereas the $\mathrm{FeO}_{6}$ octahedron has none along that direction. [See Fig. 1(a).] Such local structural characteristics are in accordance with observed magnetic properties of various hexaferrites. For instance, spins in the tetrahedral sites have strong tendencies to be oriented parallel to the $c$ axis, whereas those in the octahedral sites behave differently. The former examples include $\mathrm{BaCaFe}_{4} \mathrm{O}_{8}$ or $\mathrm{BaSrFe}_{4} \mathrm{O}_{8}$, in which $\mathrm{Fe}$ ions are found at tetrahedral sites only. ${ }^{39,40}$ In contrast, $\mathrm{Fe}$ ions in $\mathrm{BiFeO}_{3}$ occupy octahedral sites only, and its modulated magnetic structure shows no particular indication of magnetic anisotropy. ${ }^{41}$ All other hexaferrites possess both types, between which octahedral sites are found more frequently than tetrahedral sites. ${ }^{25}$ In Y-type hexaferrites, $\mathrm{S}$ blocks contain two tetrahedral sites and one octahedral site, whereas L blocks contain two tetrahedral and seven octahedral sites. Therefore, the easy-axis anisotropy of the Al-free BSZFO is ascribed to the octahedral sites that are majorities, whereas the easy-axis anisotropy emerges when they are weakened. 


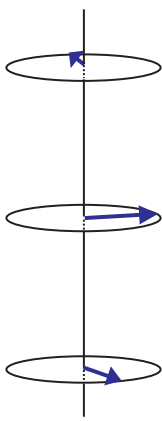

$\mathrm{PH}$

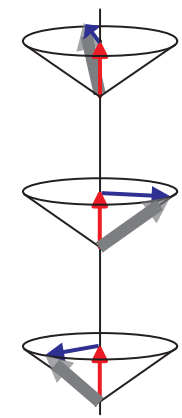

NLC

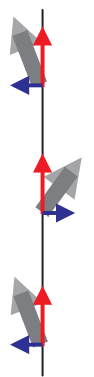

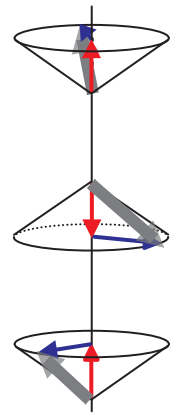

ALC

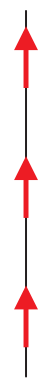

FIM

\section{Canted
AFM \\ AFM Canted
AFM}

FIG. 8. (Color online) Simplified spin structures of the magnetic phases shown in Fig. 7. L and S blocks are combined for simplicity. Red and blue arrows indicate magnetic components parallel or perpendicular to the $c$ axis, respectively. Thick gray arrows are the net magnetization.

\section{F. Analysis of the Al doping effect}

We introduce a simple magnetic Hamiltonian that can consistently explain the $\mathrm{Al}$ doping dependence of BSZFO. It includes magnetocrystalline anisotropy terms, $D_{i}$, that can be either positive (easy-plane) or negative (easy-axis) with respect to the square of the $c$-axis magnetization, such that

$$
H=\frac{1}{2} \sum_{i, j} \tilde{J}_{i j} \hat{\mu}_{i} \cdot \hat{\mu}_{j}+\sum_{i} \tilde{D}_{i}\left|\hat{\mu}_{i} \cdot \hat{z}\right|^{2} .
$$

Above, for convenience, we use $\tilde{J}_{i j}=J_{i j} \mu_{i} \mu_{j}$ for exchange interactions between the $i$ th and $j$ th spins and $\tilde{D}_{i}=$ $D_{i} \mu_{i}^{2}$ for the magnetocrystalline anisotropy of the $i$ th ion. We also assume that the spin vectors within one block, $\mathrm{L}$ or $\mathrm{S}$, are practically collinear between themselves, so only the interaction between the blocks is relevant. In other words, spins in the L blocks tilt uniformly toward the $c$ axis when necessary, whereas those in the $\mathrm{S}$ block tilt toward the opposite direction.

With the Hamiltonian defined, the PH phase can easily be found when more than two exchange interactions are considered. ${ }^{42}$ The emergence of the LC order, however, is not trivial. This is because the easy-plane anisotropy, which is necessary in order to stabilize the PH phase, will always work against the LC ordering. In fact, we realize that $\mathrm{S}$ blocks

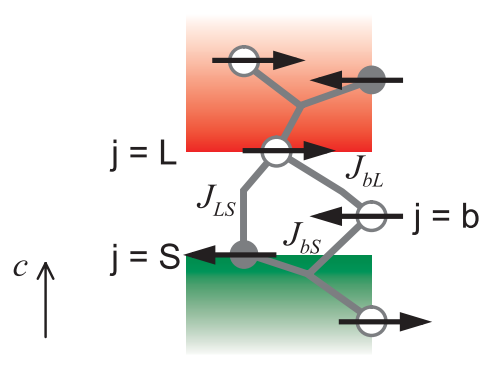

(a)

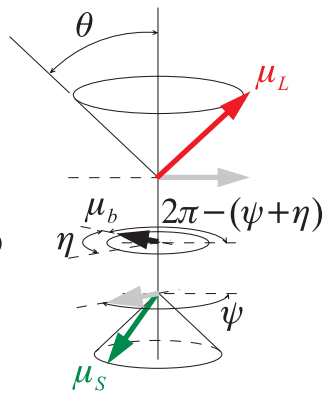

(b)
FIG. 9. (Color online) (a) A portion of the Y-type hexaferrite lattice, visualizing magnetic ions and their exchange paths near the interface between L and S blocks. Empty and filled spheres denote octahedral and tetrahedral $\mathrm{Fe}$ sites, respectively. The ion at $j=b$ serves as a bridge between $\mathrm{L}$ and $\mathrm{S}$ blocks. Oxygen ions, not shown, are located in the middle of exchange paths shown as thick solid lines. (b) Angular relations between three magnetization vectors in the NLC phase. Gray arrows are the projections onto the $a b$ plane.

include a higher number of tetrahedral sites whereas L blocks have more octahedral sites. Therefore, the net anisotropy field is probably easy-axis for the $\mathrm{S}$ blocks $\left(D_{\mathrm{S}}<0\right)$ whereas it is easy-plane for the L blocks $\left(D_{\mathrm{L}}>0\right)$. We also focus on the fact that these two blocks are bridged by octahedral $\mathrm{Fe}$ ions. [See Fig. 9(a).] Anisotropy on these bridging ions is primarily easy-plane, and therefore we assume that their spins are confined in the $a b$ planes.

Using this model, we find the equilibrium cone angle at $\sin \theta_{0}=-\left[\tilde{J}_{b \mathrm{~L}} \cos \left(\psi_{0}+\eta_{0}\right)+\tilde{J}_{b \mathrm{~S}} \cos \eta_{0}\right] / 2\left[\tilde{J}_{\mathrm{LS}}\left(\cos \psi_{0}+\right.\right.$ 1) $-\tilde{D}_{\mathrm{L}}+\tilde{D}_{\mathrm{S}}$. (See the Appendix for details.) The denominator of this solution includes both $\tilde{D}_{\mathrm{L}}$ and $\tilde{D}_{\mathrm{S}}$. When $\tilde{D}_{\mathrm{L}}$ is sufficiently large, which is the case for $x=0.0$, the $\mathrm{PH}$ phase $\left(\theta_{0}=\pi / 2\right)$ will appear because the above equation does not have a valid solution. When $\mathrm{Al}$ dopants preferentially replace octahedral sites, the average magnetic moment on these sites will be reduced. As a result, $\tilde{D}_{\mathrm{L}}$ will effectively decrease and stabilize the NLC phase with a finite cone angle $\left(\theta_{0}<\pi / 2\right)$. Further reduction of $\tilde{D}_{\mathrm{L}}$ will continue to decrease the cone angle. Al doping can also work toward the same result by a separate mechanism. Among the three exchange interactions shown in Fig. 9(a), $J_{b \mathrm{~L}}$ connects two ions at octahedral sites. Therefore, the preferential doping will more effectively reduce the strength of $\tilde{J}_{b \mathrm{~L}}$ and subsequently weaken the term $\left|\tilde{J}_{b \mathrm{~L}} \cos \left(\psi_{0}+\eta_{0}\right)\right|$ as well. Its effect on the cone angle will be similar to the reduction of $\tilde{D}_{\mathrm{L}}$.

The doping dependence of the incommensurability, $\delta$, can also be explained consistently. As shown in the Appendix, the half-pitch of the helix, $\psi=\phi / 2$, will increase if the ratio $\tilde{J}_{\mathrm{LS}} / \tilde{J}_{b \mathrm{~L}}$ is increased. Since $\tilde{J}_{b \mathrm{~L}}$ connects two octahedral sites, it will be more effectively reduced upon Al doping. [See Fig. 9(a).] Therefore, Al doping will consistently lead to the observed doping-dependent increase of $\psi$ as well as of $\delta$.

Another interesting aspect of the phase diagram is that the value of $T_{N}$ increases as a function of $\mathrm{Al}$ doping concentration, as much as $17 \%$ for $x=0.12$. This behavior may seem counterintuitive because $\mathrm{Al}$ doping will reduce the average density of magnetic moments. It can, however, be easily 
understood by considering a simple collinear FIM with two sublattices, $A$ and $B$. In the mean-field approximation, the temperature dependence of the magnetic moment on each sublattice can be written as ${ }^{43}$

$$
\begin{aligned}
& \mu_{A}=C_{A}\left(B_{a}-J_{A A} \mu_{A}-J_{A B} \mu_{B}\right) / T, \\
& \mu_{B}=C_{B}\left(B_{a}-J_{A B} \mu_{A}-J_{B B} \mu_{B}\right) / T,
\end{aligned}
$$

where $C_{i}$ is the Curie constant and $B_{a}$ is the applied field. Then the Néel temperature can be calculated for $B_{a}=0$ as follows:

$$
\begin{aligned}
T_{N}= & -\frac{1}{2}\left(J_{A A} C_{A}+J_{B B} C_{B}\right) \\
& +\sqrt{J_{A B}^{2} C_{A} C_{B}+\frac{1}{4}\left(J_{A A} C_{A}-J_{B B} C_{B}\right)^{2}} .
\end{aligned}
$$

It is easy to show that the inequality holds for $T_{N}<T_{N, 0}=$ $J_{A B} \sqrt{C_{A} C_{B}}$, in which $T_{N, 0}$ is the Néel temperature with the nearest-neighbor exchange only. It readily explains that reduction of the next-nearest-neighbor (NNN) exchange will lead to an increase in $T_{N}$. The simple model can be applied either between magnetic layers or blocks of BSZFO. Al doping again conveniently explains the preferential reduction of the NNN exchange, because the NNN exchange paths are more likely to involve two or more octahedral ions.

Finally, excessive reduction of the easy-plane anisotropy in the overdoped regime should be responsible for the deterioration of the magnetoelectric susceptibility. ${ }^{3}$ An adequate balance between the easy-axis and easy-plane anisotropy fields thus seems important in order to stabilize $90^{\circ}$ halfpitch in the field-induced magnetoelectric phases. ${ }^{26-28}$ Similar commensurate magnetic structures have been observed in other types of magnetoelectric hexaferrites, too, ${ }^{5,6}$ and they support the importance of the two coexisting anisotropy fields.

\section{SUMMARY AND CONCLUSIONS}

Using single-crystal neutron diffraction and magnetization measurements, we investigated the $x-T$ phase diagram of the magnetoelectric hexaferrite $\mathrm{Ba}_{0.7} \mathrm{Sr}_{1.3} \mathrm{Zn}_{2}\left(\mathrm{Fe}_{1-x} \mathrm{Al}_{x}\right)_{12} \mathrm{O}_{22}$ $(0 \leqslant x \leqslant 0.12)$. We found that magnetization along the $c$ axis continued to enhance throughout the studied Al doping range. Al doping also stabilized longitudinal conical phases up to $x<0.10$, beyond which commensurate ordering took place. The stabilization and subsequent collapse of the conical phases observed in this work naturally explain the doping dependence of the magnetoelectricity previously reported for $\mathrm{Ba}_{0.5} \mathrm{Sr}_{1.5} \mathrm{Zn}_{2}\left(\mathrm{Fe}_{1-x} \mathrm{Al}_{x}\right)_{12} \mathrm{O}_{22}$, particularly the appearance of the largest magnetoelectric susceptibility near $x=0.08 .^{3} \mathrm{~A}$ simple spin-exchange model could explain several Al doping effects when easy-axis and easy-plane anisotropy terms were considered separately. The results of our work demonstrate the importance of the two magnetocrystalline anisotropy terms in producing stable field-induced electric polarizations in Y-type hexaferrites.

\section{ACKNOWLEDGMENTS}

The authors are grateful to Sae Hwan Chun and Kee Hoon Kim for providing EPMA analysis and magnetoelectric measurements, as well as stimulating discussions. We also acknowledge the beam time and the scientific support provided at the TriCS diffractometer of the SINQ. This work is supported by a National Research Foundation of Korea (NRF) grant funded by the Korea government (MEST; No. 2010-0025880) and also through the Nuclear R\&D Programs (No. 2011-0019021 No. 2011-0031933).

\section{APPENDIX}

Following Momozawa et al., we consider the superexchange interactions near the interface between $\mathrm{L}$ and $\mathrm{S}$ spin blocks in Y-types hexaferrites. ${ }^{30,33}$ As shown in Fig. 9(a), the superexchange between these two blocks is either direct or goes through an additional bridging Fe ion. Since the spins in a single block are virtually collinear with each other, we treat them as a single bunch of magnetization, $\vec{\mu}_{\mathrm{L}}$ or $\vec{\mu}_{\mathrm{S}}$, respectively. The angle between these two vectors corresponds to $\psi$, a half-pitch of the incommensurate helix. Magnetization of the bridging ion located between these blocks is denoted as $\vec{\mu}_{b}$. We denote the angle between $\vec{\mu}_{\mathrm{S}}$ and $\vec{\mu}_{b}$ as $\eta$. [See Fig. 9(b).] We additionally introduce the cone angle $\theta_{i}$, by which the magnetization vector of the $i$ th block can be tilted away from the $c$ axis. In our model, we allow $\vec{\mu}_{\mathrm{L}}$ and $\vec{\mu}_{\mathrm{S}}$ vectors to be tilted toward mutually opposite directions. At the same time, $\vec{\mu}_{\mathrm{L}}$ is subject to easy-plane anisotropy whereas $\vec{\mu}_{\mathrm{S}}$ is subject to easy-axis anisotropy for the reason discussed previously. The bridging $\vec{\mu}_{b}$ is fixed within the $a b$ planes.

For simplicity, the reduced forms of the Heisenberg exchange and planar anisotropy constants are used, such that $\tilde{J}_{i j}=J_{i j} \mu_{i} \mu_{j}$ and $\tilde{D}_{i}=D_{i} \mu_{i}^{2}$, respectively. The total magnetic energy for the NLC phase can then be written as

$$
\begin{aligned}
E / N= & \tilde{J}_{\mathrm{LS}}\left(\sin \theta_{\mathrm{L}} \sin \theta_{\mathrm{S}} \cos \psi-\cos \theta_{\mathrm{L}} \cos \theta_{\mathrm{S}}\right) \\
& +\tilde{J}_{b \mathrm{~L}} \sin \theta_{\mathrm{L}} \cos (\psi+\eta) \\
& +\tilde{J}_{b \mathrm{~S}} \sin \theta_{\mathrm{S}} \cos \eta \\
& +\tilde{D}_{\mathrm{L}} \cos ^{2} \theta_{\mathrm{L}}-\tilde{D}_{\mathrm{S}} \cos ^{2} \theta_{\mathrm{S}}
\end{aligned}
$$

For further simplicity, we will consider only the case of $\theta_{\mathrm{L}}=\theta_{\mathrm{S}}=\theta$. By taking partial derivatives of the total energy,

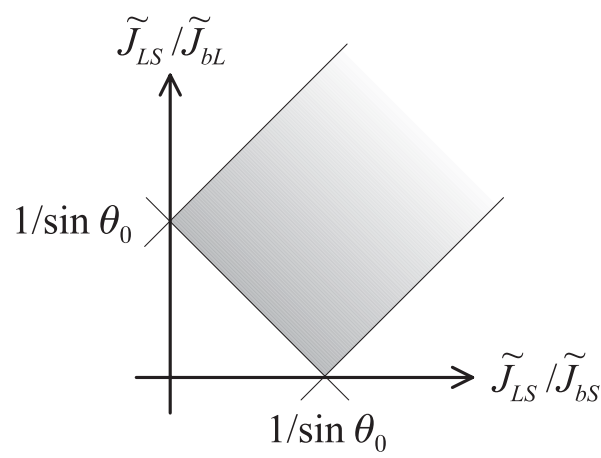

FIG. 10. An incommensurate helix can become stable if the ratios between the exchange terms fall within in the grey-shaded area. 
we obtain the minimum energy configuration in terms of $\psi$ and $\eta$, respectively, as functions of the equilibrium cone angle, $\theta_{0}$ :

$$
\begin{aligned}
& \cos \psi_{0}=\frac{\tilde{J}_{b \mathrm{~S}} \tilde{J}_{b \mathrm{~L}}}{2 \tilde{J}_{\mathrm{LS}}^{2}}\left[\frac{1}{\sin ^{2} \theta_{0}}-\left(\frac{\tilde{J}_{\mathrm{LS}}}{\tilde{J}_{b \mathrm{~S}}}\right)^{2}-\left(\frac{\tilde{J}_{\mathrm{LS}}}{\tilde{J}_{b \mathrm{~L}}}\right)^{2}\right], \\
& \cos \eta_{0}=\frac{\tilde{J}_{b \mathrm{~L}}}{2 \tilde{J}_{\mathrm{LS}} \sin \theta_{0}}\left[\left(\frac{\tilde{J}_{\mathrm{LS}} \sin \theta_{0}}{\tilde{J}_{b \mathrm{~S}}}\right)^{2}-\left(\frac{\tilde{J}_{\mathrm{LS}} \sin \theta_{0}}{\tilde{J}_{b \mathrm{~L}}}\right)^{2}-1\right] .
\end{aligned}
$$

An incommensurate helix can stabilize for $-1<\cos \psi_{0}<$ 1 and $-1<\cos \eta_{0}<1$. The ranges of exchange constants satisfying these constraints are displayed in Fig. 10.

The equilibrium value of the cone angle, $\theta_{0}$, can also be obtained as a function of the above two angles:

$$
\sin \theta_{0}=\frac{-\tilde{J}_{b \mathrm{~L}} \cos \left(\psi_{0}+\eta_{0}\right)-\tilde{J}_{b \mathrm{~S}} \cos \eta_{0}}{2\left[\tilde{J}_{\mathrm{LS}}\left(\cos \psi_{0}+1\right)-\tilde{D}_{\mathrm{L}}+\tilde{D}_{\mathrm{S}}\right]}
$$

The actual value of $\theta_{0}$ may be obtained independently by solving the third-order equation as follows:

$$
\begin{gathered}
A \sin ^{3} \theta_{0}+B \sin ^{2} \theta_{0}+C \sin \theta_{0}+D=0, \\
A=\tilde{J}_{\mathrm{LS}}^{3}\left(\tilde{J}_{b \mathrm{~L}}^{2}-\tilde{J}_{b \mathrm{~S}}^{2}\right), \\
B=2 \tilde{J}_{\mathrm{LS}} \tilde{J}_{b \mathrm{~L}} \tilde{J}_{b \mathrm{~S}}^{2}\left(\tilde{J}_{\mathrm{LS}}+\tilde{D}_{\mathrm{S}}-\tilde{D}_{\mathrm{L}}\right), \\
C=-\tilde{J}_{\mathrm{LS}} \tilde{J}_{b \mathrm{~L}}^{2} \tilde{J}_{b \mathrm{~S}}^{2},=-\tilde{J}_{b \mathrm{~L}}^{2} \tilde{J}_{b \mathrm{~S}}^{3} .
\end{gathered}
$$

The general solution of the above third-order equation is lengthy and in no way didactic, so we will not write it out in this appendix. Nevertheless, in the following we can prove that a real solution for $\theta_{0}$ does exist. The NLC phase will stabilize if Eq. (A3) satisfies the constraint $0<\sin \theta_{0}<1$. This inequality can be rewritten as $0<-\tilde{J}_{b \mathrm{~L}} \cos \left(\psi_{0}+\eta_{0}\right)-$ $\tilde{J}_{b \mathrm{~S}} \cos \eta_{0}<2\left[\tilde{J}_{\mathrm{LS}}\left(\cos \psi_{0}+1\right)-\tilde{D}_{\mathrm{L}}+\tilde{D}_{\mathrm{S}}\right]$. The first part of the inequality can be easily met if $\pi / 2<\psi_{0}+\eta_{0}<3 \pi / 2$ and $\tilde{J}_{b \mathrm{~S}}<<\tilde{J}_{b \mathrm{~L}}$. The second part can then be rearranged as $-\tilde{J}_{b \mathrm{~L}} \cos \left(\psi_{0}+\eta_{0}\right)-\tilde{J}_{b \mathrm{~S}} \cos \eta_{0}+2 \tilde{D}_{\mathrm{L}}<2\left[\tilde{J}_{\mathrm{LS}}\left(\cos \psi_{0}+\right.\right.$ 1) $\left.+\tilde{D}_{\mathrm{S}}\right]$. This inequality can be satisfied if $\tilde{D}_{\mathrm{L}}$ becomes sufficiently smaller than $\tilde{D}_{\mathrm{S}}$. It is therefore important to have a substantially strong easy-axis anisotropy in the first place. *jaehc@korea.ac.kr

${ }^{1}$ T. Kimura, G. Lawes, and A. P. Ramirez, Phys. Rev. Lett. 94, 137201 (2005).

${ }^{2}$ S. Ishiwata, Y. Taguchi, H. Murakawa, Y. Onose, and Y. Tokura, Science 319, 1643 (2008).

${ }^{3}$ S. H. Chun, Y. S. Chai, Y. S. Oh, D. Jaiswal-Nagar, S. Y. Haam, I. Kim, B. Lee, D. H. Nam, K.-T. Ko, J.-H. Park, J.-H. Chung, and K. H. Kim, Phys. Rev. Lett. 104, 037204 (2010).

${ }^{4}$ Y. Kitagawa, Y. Hiraoka, T. Honda, T. Ishikura, H. Nakamura, and T. Kimura, Nat. Mater. 9, 797 (2010).

${ }^{5}$ Y. Tokunaga, Y. Kaneko, D. Okuyama, S. Ishiwata, T. Arima, S. Wakimoto, K. Kakurai, Y. Taguchi, and Y. Tokura, Phys. Rev. Lett. 105, 257201 (2010).

${ }^{6}$ M. Soda, T. Ishikura, H. Nakamura, Y. Wakabayashi, and T. Kimura, Phys. Rev. Lett. 106, 087201 (2011).

${ }^{7}$ K. Okumura, T. Ishikura, M. Soda, T. Asaka, H. Nakamura, Y. Wakabayashi, and T. Kimura, Appl. Phys. Lett. 98, 212504 (2011).

${ }^{8}$ S. H. Chun, Y. S. Chai, B.-G. Jeon, H. J. Kim, Y. S. Oh, I. Kim, H. Kim, B. J. Jeon, S. Y. Haam, J.-Y. Park, S. H. Lee, J.-H. Chung, J.-H. Park, and K. H. Kim (unpublished).

${ }^{9}$ H. Katsura, N. Nagaosa, and A. V. Balatsky, Phys. Rev. Lett. 95, 057205 (2005).

${ }^{10}$ W. Eerenstein, N. D. Mathur, and J. F. Scott, Nature (London) 442, 759 (2006).

${ }^{11}$ S.-W. Cheong and M. Mostovoy, Nat. Mater. 6, 13 (2007).

${ }^{12}$ A. B. Harris, T. Yildirim, A. Aharony, and O. Entin-Wohlman, Phys. Rev. B 73, 184433 (2006).

${ }^{13}$ M. Mostovoy, Phys. Rev. Lett. 96, 067601 (2006).

${ }^{14}$ M. Kenzelmann, A. B. Harris, S. Jonas, C. Broholm, J. Schefer, S. B. Kim, C. L. Zhang, S.-W. Cheong, O. P. Vajk, and J. W. Lynn, Phys. Rev. Lett. 95, 087206 (2005).

${ }^{15}$ G. Lawes, A. B. Harris, T. Kimura, N. Rogado, R. J. Cava, A. Aharony, O. Entin-Wohlman, T. Yildrim, M. Kenzelmann, C. Broholm, and A. P. Ramirez, Phys. Rev. Lett. 95, 087205 (2005).
${ }^{16}$ L. C. Chapon, P. G. Radaelli, G. R. Blake, S. Park, and S.-W. Cheong, Phys. Rev. Lett. 96, 097601 (2006).

${ }^{17}$ K. Taniguchi, N. Abe, T. Takenobu, Y. Iwasa, and T. Arima, Phys. Rev. Lett. 97, 097203 (2006).

${ }^{18}$ Y. Yamasaki, H. Sagayama, T. Goto, M. Matsuura, K. Hirota, T. Arima, and Y. Tokura, Phys. Rev. Lett. 98, 147204 (2007).

${ }^{19}$ T. Kimura, T. Goto, H. Shintani, K. Ishizaka, T. Arima, and Y. Tokura, Nature (London) 426, 55 (2003).

${ }^{20}$ N. Hur, S. Park, P. A. Sharma, J. S. Ahn, S. Guha, and S-W. Cheong, Nature (London) 429, 392 (2004).

${ }^{21}$ M. Gajek, M. Bibes, S. Fusil, K. Bouzehouane, J. Fontcuberta, A. Barthélémy, and A. Fert, Nat. Mater. 6, 296 (2007).

${ }^{22}$ H. Sagayama, K. Taniguchi, N. Abe, T.-H. Arima, M. Soda, M. Matsuura, and K. Hirota.Phys. Rev. B 77, 220407(R) (2008).

${ }^{23}$ Y.-H. Chu, L. W. Martin, M. B. Holcomb, M. Gajek, S.-J. Han, Q. He, N. Balke, C.-H. Yang, D. Lee, W. Hu, Q. Zhan, P.-L. Yang, A. Fraile-Rodríguez, A. Scholl, S. X. Wang, and R. Ramesh, Nat. Mater. 7, 478 (2008).

${ }^{24}$ S. Valencia, A. Crassous, L. Bocher, V. Garcia, X. Moya, R. O. Cherifi, C. Deranlot, K. Bouzehouane, S. Fusil, A. Zobelli, A. Gloter, N. D. Mathur, A. Gaupp, R. Abrudan, F. Radu, A. Barthélémy, and M. Bibes, Nat. Mater. 10, 753 (2011).

${ }^{25}$ J. Smit and H. P. J. Wijn, Ferrites (Philips Technical Library, Eindhoven, 1959), Chap. IX.

${ }^{26}$ H. Sagayama, K. Taniguchi, N. Abe, T.-H. Arima, Y. Nishikawa, S.-I. Yano, Y. Kousaka, J. Akimitsu, M. Matsuura, and K. Hirota, Phys. Rev. B 80, 180419(R) (2009).

${ }^{27}$ S. Ishiwata, D. Okuyama, K. Kakurai, M. Nishi, Y. Taguchi, and Y. Tokura, Phys. Rev. B 81, 174418 (2010).

${ }^{28}$ H. B. Lee, Y.-S. Song, J.-H. Chung, S. H. Chun, Y. S. Chai, K. H. Kim, M. Reehuis, K. Prokeš, and S. Mat' aš, Phys. Rev. B 83, 144425 (2011). 
${ }^{29}$ T. M. Perekalina, V. A. Sizov, R. A. Sizov, I. I. Yamzin, and R. A. Voskanyan, Soviet Physics JETP 25, 266 (1967).

${ }^{30}$ S. Utsumi, D. Yoshiba, and N. Momozawa, J. Phys. Soc. Jpn. 76, 034704 (2007).

${ }^{31}$ N. Momozawa, Y. Yamaguchi, H. Takei, and M. Mita, J. Phys. Soc. Jpn. 54, 771 (1985).

${ }^{32}$ N. Momozawa and Y. Yamaguchi, J. Phys. Soc. Jpn. 62, 1292 (1993).

${ }^{33}$ N. Momozawa, Y. Nagao, S. Utsumi, M. Abe, and Y. Yamaguchi, J. Phys. Soc. Jpn. 70, 2724 (2001).

${ }^{34}$ N. Momozawa and M. Mita, J. Cryst. Growth 83, 403 (1987).

${ }^{35}$ J. Schefer, M. Könnecke, A. Murasik, A. Czopnik, Th. Strässle, P. Keller, and N. Schlumpf, Physica B 276-278, 168 (2000).

${ }^{36}$ W. E. Fischer, Physica B 234-236, 1202 (1997).
${ }^{37}$ In our previous report, ${ }^{28}$ we used the incommensurability such that $k_{2}=(0,0, \delta)$. In the current work, we instead use $k_{2}=(0,0,3 \delta)$ following the convention of Momozawa et al. ${ }^{31}$

${ }^{38}$ See Supplemental Material at http://link.aps.org/supplemental/ 10.1103/PhysRevB.85.064402 for the figures in an enlarged format.

${ }^{39}$ Y. Abbas, F. Mostafa, and M. Fayek, J. Phys. Chem. Solids 43, 973 (1982).

${ }^{40}$ Y. Abbas, F. Mostafa, and M. Fayek, Acta Crystallogr. Sect. B 39, 1 (1983).

${ }^{41}$ I. Sosnowska, T. Peterlinneumaier, and E. Steichele, J. Phys. Solid State Phys. 15, 4835 (1982).

${ }^{42}$ D. Khomskii, Basic Aspects of the Quantum Theory of Solids (Cambridge University Press, Cambridge, UK, 2010), Chap. 6.

${ }^{43}$ C. Kittel, Introduction to Solid State Physics, 8th ed. (Wiley, Hoboken, NJ, USA, 2005), Chap. 12. 